\title{
Effects of a Multicomponent Exercise Program on Spatiotemporal Gait Parameters, Risk of Falling and Physical Activity in Dementia Patients
}

\author{
Anaïck Perrochon ${ }^{a} \quad$ Achille E. Tchalla $^{a} b \quad$ Joelle Bonis ${ }^{a} \quad$ Florian Perucaud $^{a}$ \\ Stéphane Mandigout ${ }^{a}$ \\ ${ }^{a}$ HAVAE, EA 6310, Université de Limoges, and ${ }^{b} \mathrm{CHU}$ Limoges, Pôle Clinique Médicale et \\ Gérontologie Clinique, Service de Médecine Gériatrique, Limoges, France
}

Key Words

Multicomponent exercise · Gait parameters · Risk of falling · Dementia

\begin{abstract}
Background: Exercise programs are presumed to rehabilitate gait disorders and to reduce the risk of falling in dementia patients. This study aimed to analyze the specific effects of multicomponent exercise on gait disorders and to determine the association between gait impairments and the risk of falling in dementia patients before and after intervention. Methods: We conducted an 8-week multicomponent exercise program in 16 dementia patients (age $86.7 \pm$ 5.4 years). All participants were assessed several times for gait analysis (Locométrix ${ }^{\circledR}$ ), Tinetti score and physical activity (Body Media SenseWear ${ }^{\circledR}$ Pro armband). Results: After 8 weeks of the exercise program, the mean gait speed was $0.12 \mathrm{~m} / \mathrm{s}$ faster than before the intervention $(0.55 \pm 0.17$ vs. $0.67 \pm 0.14 \mathrm{~m} / \mathrm{s})$. The multicomponent exercise program improved gait performance and Tinetti score $(p<0.05)$. Gait performance (gait speed, stride length) was correlated with the Tinetti score $(p<0.05)$. Conclusion: Analysis of spatiotemporal gait parameters using an accelerometer method provided a quick and easy tool to estimate the benefits of an exercise program and the risk of falling.

(C) 2015 S. Karger AG, Basel
\end{abstract}

\section{Introduction}

In addition to cognitive decline, mobility and balance impairments are common features of dementia [1]. Indeed, gait disorders are more prevalent in dementia than in normal aging, and dementia-related gait changes usually lead to falls [2-4]. Patients with dementia are at 
Perrochon et al.: Effects of a Multicomponent Exercise Program on Spatiotemporal Gait Parameters, Risk of Falling and Physical Activity in Dementia Patients

high risk of falling and suffer from mobility limitations, but intensive physical exercise may improve mobility [5-7] and the clinically meaningful gait variables [8-10], and therefore reduce the risk of falling $[11,12]$. A systematic review demonstrated the positive impact of different exercise programs (e.g. resistance exercises, aerobic exercises, functional daily living exercises and dancing) on daily activities, autonomy, quality of life, balance and walking in dementia patients [13]. Thus, an exercise program may represent a model to reduce the risk of falling and rehabilitate gait impairment in dementia patients.

Many authors have noted that exercise programs open opportunities for community health to provide prevention and to reduce the rate and risk of falling in the elderly [14-16]. However, the effectiveness of exercise programs in fall prevention in dementia patients is limited $[3,17]$. Only a few studies have shown a positive effect on postural control and the risk of falling in this population $[11,12]$. The efficiency of an exercise program on mobility and physical functioning has been demonstrated recently in both institutionalized $[5,9,18]$ and community-dwelling dementia patients [6,7]. More precisely, the effectiveness of exercise for improving spatiotemporal gait parameters in those with cognitive impairment has been demonstrated recently [8-10]. Few studies have shown that intensive exercise is practicable and improves the clinically meaningful gait variables in dementia patients [10], even at an early stage of dementia [8]. Thus, an exercise program appears to be a solution that enhances physical function and gait parameters among elderly with cognitive impairments, but the impact of exercise programs on balance is more debatable.

After considering the benefits of maintaining gait performance through an exercise program in dementia patients, it is important to determine whether gait parameter impairments are associated with the risk factors of falling in this population. Recent studies have shown that there is a link between gait variability, cognitive dysfunction and the risk of falls [13]. Previous studies have highlighted that some aspects of gait, such as gait parameters and walking conditions, could predict an increased risk of falling in elderly with cognitive impairments $[2,3]$. Gait changes are often too discrete to be detected by clinical observation alone. Thus, many technologies have been developed to assess spatiotemporal gait parameters (e.g. optoelectronic systems, the electronic walkway system and triaxial accelerometer devices) $[19,20]$. Among the triaxial accelerometer systems, Locométrix ${ }^{\circledR}$ is a very simple and economical gait analysis device with significant reliability [21, 22]. Moreover, Locométrix ${ }^{\circledR}$ has already been used to define gait profiles at different stages of dementia [23, 24]. It seems that accelerometer systems, such as Locométrix ${ }^{\circledR}$ or Dynaport ${ }^{\circledR}$, allow the study of the relationship between gait performance and the risk of falling [20,22]. Besides, this tool was used to reveal that some specific gait markers are strong predictors of the risk of a first fall [25].

The main objective of this study was to determine the effects of multicomponent exercise on gait parameters, the risk of falling and physical activity in dementia patients. The second aim was to explore the link between spatiotemporal gait parameters and the risk of falling in these patients with a simple gait analysis device.

\section{Materials and Methods}

\section{Participants}

Sixteen participants aged $>80$ years with cognitive impairments participated in the study. Among these people, 8 participants had fallen at least once. Participants were recruited consecutively from either a geriatric hospital (Alzheimer's disease hospital unit) or a nursing home. The diagnosis of Alzheimer's disease or other form of dementia was performed by geriatricians according to the Diagnostic and Statistical Manual of Mental Disorders, 4th edition 
Perrochon et al.: Effects of a Multicomponent Exercise Program on Spatiotemporal Gait Parameters, Risk of Falling and Physical Activity in Dementia Patients

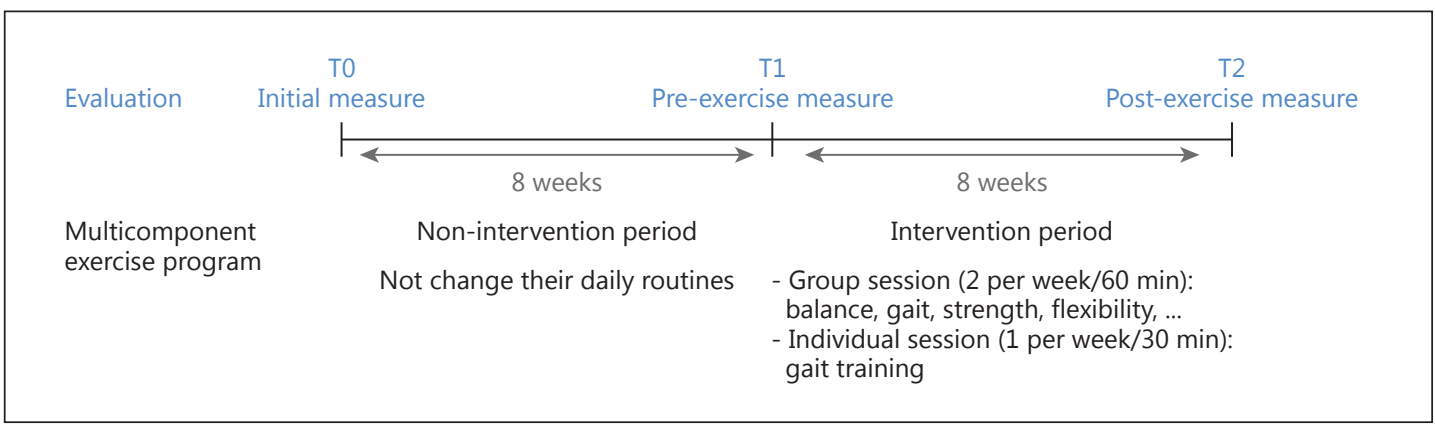

Fig. 1. Timeline and structure of the study design.

(DSM-IV) criteria and/or the Mini-Mental State Examination (MMSE) score $(<23)$ [26]. MMSE provides a brief test that quantitatively assesses the severity of cognitive impairment of participants. The mean score of our population was $15.5 \pm 5$.9. The etiology of dementia was not clearly established for 4 patients.

To be eligible for inclusion, participants were required to have lived in institutional care for at least 2 months, to be able to rise from a chair and to walk at least $10 \mathrm{~m}$ with or without the assistance of a cane, and to have an MMSE score $<23$. Participants presented a sufficient level of autonomy to move by themselves and were recruited on their capability to understand instructions and to follow an adapted physical activity program. Exclusion criteria included a history of major psychiatric illness (e.g. schizophrenia or bipolar disorder) or other serious neurological or musculoskeletal diagnoses (e.g. Parkinson's disease or stroke) that could influence posture and/or gait. Written consent was obtained from patients, caregivers or legal guardians.

\section{Design}

In this study there were two different periods of 8 weeks (a non-intervention period and an intervention period) (fig. 1). In the first period, participants did not change their daily routines and no activities were proposed (non-intervention). In the second period, we proposed a multicomponent exercise program (intervention) to all participants. The 8-week multicomponent exercise program consisted of two group sessions and one individual session per week based on endurance walking, balance, flexibility, strength and gait exercises. The duration of the group sessions was about $60 \mathrm{~min}$ and the duration of the individual session was $30 \mathrm{~min}$.

\section{Group Sessions}

The group sessions were divided into three parts: the first part consisted of $10 \mathrm{~min}$ of contact and a stretching warm-up, the second part consisted of $40 \mathrm{~min}$ of multicomponent exercise (strength, balance and gait exercises), and the third part consisted of 10 min of flexibility and relaxation.

In the second part, the program consisted of moderate intensity exercises of progressive resistance strength training of the lower extremities. We alternated between elastic bands (e.g. theraband) and functional exercises (sitting down, standing up from a sitting position on a chair, lateral elevation of the legs in a standing position, climbing stairs). Postural balance exercises, such as tandem walking and side walking on balance boards using cones and hoops 
on the ground, one- or two-leg balance exercises, and rising on the toes were also included in the sessions. In addition, participants performed combined balance and walking exercises (e.g. circuit exercise including stair stepping and endurance walking).

\section{Individual Session}

Once a week the individual session focused on gait exercises. When participants were stable in basic walking, the complexity and challenge of the tasks was increased using goaloriented progressively difficult stepping, then modification of walking speed and amplitude (e.g. narrowing oval width) and zigzagging. Finally, gait exercises included dual-task walking (e.g. walking whilst conducting a conversation, walking while counting, walking while carrying a glass of water, or walking while bouncing a ball).

A geriatric rehabilitation specialist supervised the exercises and a trained physiotherapist conducted the sessions. To enhance adherence, the exercise program began at a light intensity and was adapted to the capability of the participants so that they could repeat the exercises easily. We explained the exercise program using simple sentences and exercises so that the patients understood the instructions.

\section{Outcome Measurements}

Spatiotemporal gait analysis and the Tinetti balance assessment were measured several times (fig. 1): at the beginning of the non-intervention period (T0, initial measurements), before the intervention period (T1, pre-exercise measurements), and after the intervention period at the end of the 8-week multicomponent exercise program (T2, post-exercise measurements). Finally, physical activity was assessed during the non-intervention and intervention periods.

\section{Gait Parameters}

Participants were asked to walk at a comfortable pace along a straight corridor $20 \mathrm{~m}$ long. All participants wore their usual walking shoes, avoiding high heels or hard-soled shoes. Gait parameters were recorded at $100 \mathrm{~Hz}$ using a Locométrix ${ }^{\circledR}$ triaxial accelerometer (Centaure Metrix, Evry, France) [23, 24, 27, 28], which features a system comprising two perpendicularly arranged accelerometers placed near the subject's center of gravity by an elastic belt (at the L3-L4 intervertebral space). The sensor weighed $20 \mathrm{~g}$ and was composed of three accelerometers placed perpendicularly to each other and housed in a small box $(40 \times 18 \times 18 \mathrm{~mm})$ [21].

The following variables were calculated during the walking task: (1) Gait speed (m/s) was measured using a timing line. (2) Stride frequency ( $\mathrm{Hz}$ ) was calculated from the craniocaudal acceleration measurements and following the application of a fast Fourier transform. It was normalized according to the height of each participant. (3) Stride length (m) was deduced from the equation (stride length = speed/stride frequency). (4) The symmetry index (arbitrary units) was calculated. It described the similarity of left and right craniocaudal acceleration, which is the integral of the power spectrum of density calculated from the fast Fourier transform of the craniocaudal acceleration signal. The symmetry index is independent of fluctuations in the successive craniocaudal movements of each limb and it is dimensionless. Symmetry was calculated over all the steps of a sample by an autocorrelation function of the signal craniocaudal acceleration. A value $>200$ was considered normal. (5) The regularity index (arbitrary units) measured the similarity (in terms of duration and amplitude) of the shape of the craniocaudal acceleration curves from one step to another. A value $>300$ was considered normal. Symmetry and regularity may be independent of age and gender. 
Perrochon et al.: Effects of a Multicomponent Exercise Program on Spatiotemporal Gait Parameters, Risk of Falling and Physical Activity in Dementia Patients

\section{Tinetti Balance Scale}

We assessed all participants' gait and balance abilities using the simple, easily administered Tinetti scale [27]. The resulting scores range from 0 to 28 . When the score is $\leq 18$, the fall risk is considered high, when the score is between 18 and 24, the fall risk is considered moderate, and when the score is $\geq 24$, the fall risk is considered low [27]. Also, the Tinetti score is indirectly associated with the risk of falling.

\section{Physical Activity}

Analysis of energy expenditure for physical activity during daily life was performed using a triaxial accelerometer (Body Media SenseWear ${ }^{\circledR}$ Pro armband). Indeed, accelerometers can quantify the intensity, frequency, duration and total volume of activity completed and give an indication of physical activity. It assesses energy expenditure for physical activity and is expressed as the metabolic equivalent (MET) [28]. Energy expenditure was measured every day before (non-intervention) and during (intervention) the 8-week multicomponent exercise program. The accelerometer was worn on the wrist for $12 \mathrm{~h}$ per day (from 9 a.m. to 9 p.m.) in the geriatric hospital or the nursing home.

\section{Statistical Analysis}

The Shapiro-Wilk test was used to test the normal distribution of each parameter. Repeated measurements were studied by analysis of variance (repeated ANOVA) (T0 vs. T1 vs. T2), and significant differences were determined by a post hoc Fisher's test. Spearman's rank correlation test was used to study the link between spatiotemporal gait parameters and the Tinetti score. Finally, the Wilcoxon test was used to analyze the effects of the exercise program on physical activity. Statistical analysis was performed using Statistica software version 9 (StatSoft Inc., Tulsa, Okla., USA). The significance level was set at $\mathrm{p}<0.05$.

\section{Results}

The mean anthropometric parameters of the population were age $86.7 \pm 5.4$ years, weight $64.7 \pm 13.5 \mathrm{~kg}$, height $155.1 \pm 5.6 \mathrm{~cm}$ and body mass index $26.91 \pm 5.65$.

\section{Effects of the Multicomponent Exercise Program on Outcome Measurements}

First, gait speed increased significantly between before (T0 and T1) and after intervention (T2) $(\mathrm{p}<0.05)$ (table 1). Indeed, after 8 weeks of the exercise program (T2), the mean gait speed was $0.12 \mathrm{~m} / \mathrm{s}$ faster than at initial measurement (T0) and pre-exercise measurement (T1) $[0.55 \pm 0.17$ (T0) vs. $0.67 \pm 0.14 \mathrm{~m} / \mathrm{s}$ (T2), $\mathrm{p}<0.05 ; 0.55 \pm 0.16$ (T1) vs. $0.67 \pm 0.14 \mathrm{~m} / \mathrm{s}$ (T2), $\mathrm{p}<0.05$ ] (table 1). However, gait speed did not change significantly between T0 and T1 ( $p>0.05$ ). Among the other gait parameters, stride length, stride frequency and the symmetry index increased significantly at T2 compared to the baseline values at T0 and T1 $(p<0.05)$, but there was no significant difference between T0 and T1 ( $>>0.05)$ (table 1). Finally, there was no significant difference for regularity index between T0 and T1 ( $p>0.05)$.

Second, the results showed differences in the Tinetti score before (T0 and T1) and after intervention ( $\mathrm{T} 2)(\mathrm{p}<0.05)$ (table 1). Indeed, post hoc analysis revealed that the Tinetti score at T2 (23.7 \pm 4.2$)$ increased significantly from T0 $(22.1 \pm 5.7, \mathrm{p}<0.05)$ and $\mathrm{T} 1(22.4 \pm 5.5, \mathrm{p}<$ $0.05)$, but no significant difference was observed between T0 and T1 ( $p>0.05$ ).

Third, energy expenditure assessed by the Body Media SenseWear ${ }^{\circledR}$ Pro armband did not reveal any differences between the metabolic cost of physical activity of the 8-week exercise 
Table 1. Comparison of spatiotemporal gait parameters and Tinetti score between three measurements (T0, T1, T2) by repeated ANOVA

\begin{tabular}{l|l}
\hline Dement Geriatr Cogn Disord Extra 2015;5:350-360 \\
\hline DOI: 10.1159/000435772 & $\begin{array}{l}\text { @ 2015 S. Karger AG, Basel } \\
\text { www.karger.com/dee }\end{array}$ \\
\hline
\end{tabular}

Perrochon et al.: Effects of a Multicomponent Exercise Program on Spatiotemporal Gait Parameters, Risk of Falling and Physical Activity in Dementia Patients

\begin{tabular}{lrrr}
\hline & T0 & \multicolumn{1}{c}{ T1 } & \multicolumn{1}{c}{ T2 } \\
\hline Gait parameters & & & \\
Gait speed, m/s & $0.55 \pm 0.17^{*}$ & $0.55 \pm 0.16^{\#}$ & $0.67 \pm 0.14$ \\
Stride length, m & $0.74 \pm 0.12^{*}$ & $0.77 \pm 0.16^{\#}$ & $0.82 \pm 0.13$ \\
Stride frequency, Hz & $0.73 \pm 0.13^{*}$ & $0.72 \pm 0.14^{\#}$ & $0.81 \pm 0.07$ \\
Regularity index, a.u. & $73.2 \pm 41.6^{*}$ & $73.1 \pm 35.5^{\#}$ & $89.6 \pm 44.0$ \\
Symmetry index, a.u. & $144.6 \pm 38.8$ & $154.4 \pm 51.3$ & $208.0 \pm 58.9$ \\
\hline Clinical test & & & \\
Tinetti score & $22.1 \pm 5.7^{*}$ & $22.4 \pm 5.5^{\#}$ & $23.7 \pm 4.2$ \\
\hline
\end{tabular}

Figures are given as mean \pm standard deviation.

a.u. = Arbitrary units.

* Significantly different between T0 and T2. * Significantly different between $\mathrm{T} 1$ and $\mathrm{T} 2$.

program and without it $(1.11 \pm 1.15$ MET for non-intervention vs. 1.15 \pm 0.22 MET for intervention, $p>0.05$ ).

\section{Relationship between Gait Parameters and the Tinetti Score}

We observed a significant positive correlation between gait speed and the Tinetti score at T0 $(r=0.56, p<0.05), T 1(r=0.56, p<0.05)$ and T2 ( $=0.50, p<0.05)$ (fig. 2a). Similarly, stride length was associated with the Tinetti score in the three measurements (T0: $r=0.70$; $\mathrm{T} 1 \mathrm{r}=0.58 ; \mathrm{T} 2: \mathrm{r}=0.58 ; \mathrm{p}<0.05$ ) (fig. $2 \mathrm{~b}$ ). Among the others gait parameters, stride frequency and symmetry were not associated with the Tinetti score $(\mathrm{p}>0.05)$.

\section{Discussion}

The present study aimed to define the specific effect of multicomponent exercise on gait parameters, risk of falling and physical activity in dementia patients. Secondly, it aimed to determine the link between spatiotemporal gait parameters and the risk of falling in this population.

\section{Effects of the Multicomponent Exercise Program on Outcome Measurements}

The main result showed that a multicomponent exercise program improved gait parameters and decreased the risk of falling in dementia patients. In the literature, multicomponent exercise has been shown to improve gait speed [8-10], stride length [8-10], cadence and the double support time $[9,10]$. In this study, the improved gait parameters included gait speed, cadence, stride length and the symmetry index, but not the regularity index. The effect of exercise on gait speed obtained in this study $(0.12 \mathrm{~m} / \mathrm{s})$ was similar to that found in a recent meta-analysis (mean difference $0.06 \mathrm{~m} / \mathrm{s}$ ) [13], but this value was less significant than the results of several recent studies (gait speed increase from 0.18 to $0.28 \mathrm{~m} / \mathrm{s}$ ) [8-10]. Moreover, the symmetry index score also increased and our patients reached the normal value (score $>200$ ) [21]. Our exercise program significantly increased the average score of the Tinetti test, but this improvement was shown specifically for participants whose score indicated a high or moderate risk of falling before the 8-week exercise program. Indeed, 4 participants out of 8 moved from high to moderate risk of falling, and 1 participant out of 8 moved from moderate to low risk of falling. These improvements are in agreement with the work of Santana-Sosa et 
Perrochon et al.: Effects of a Multicomponent Exercise Program on Spatiotemporal

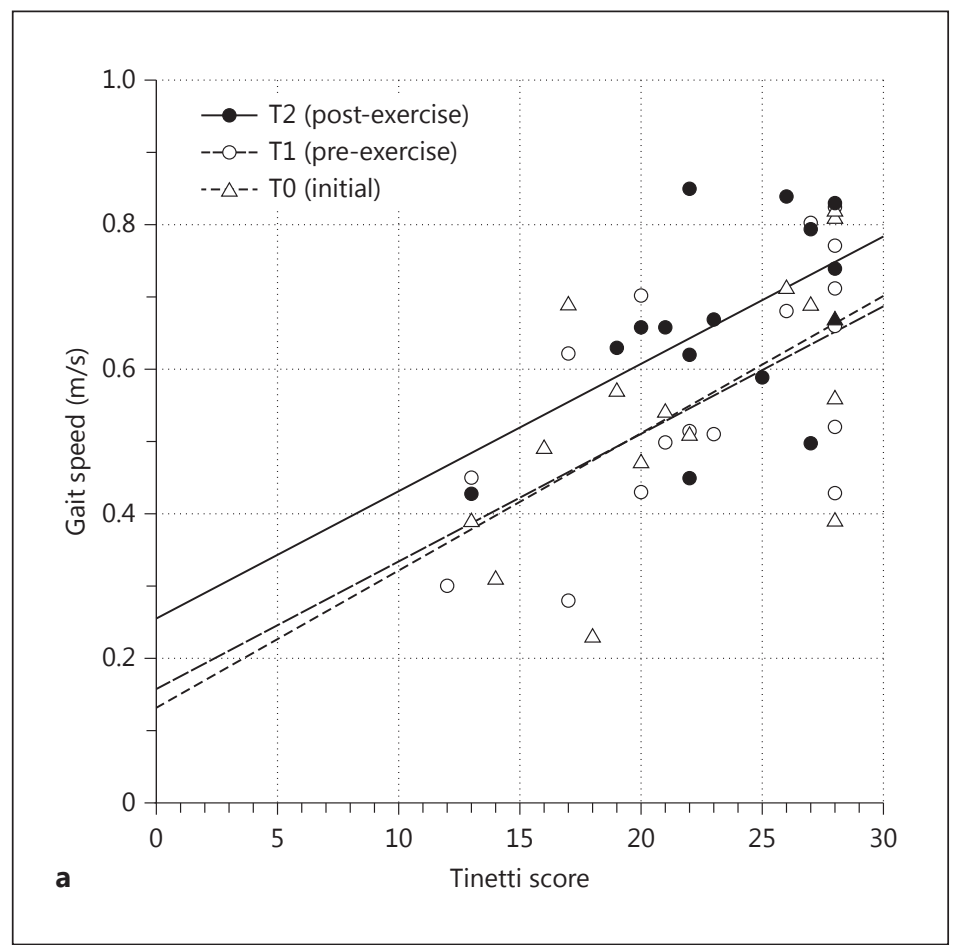

Fig. 2. a Correlation between gait speed and the Tinetti score at T0, $\mathrm{T} 1$ and $\mathrm{T} 2$. b Correlation between stride length and the Tinetti score at T0, T1 and T2.

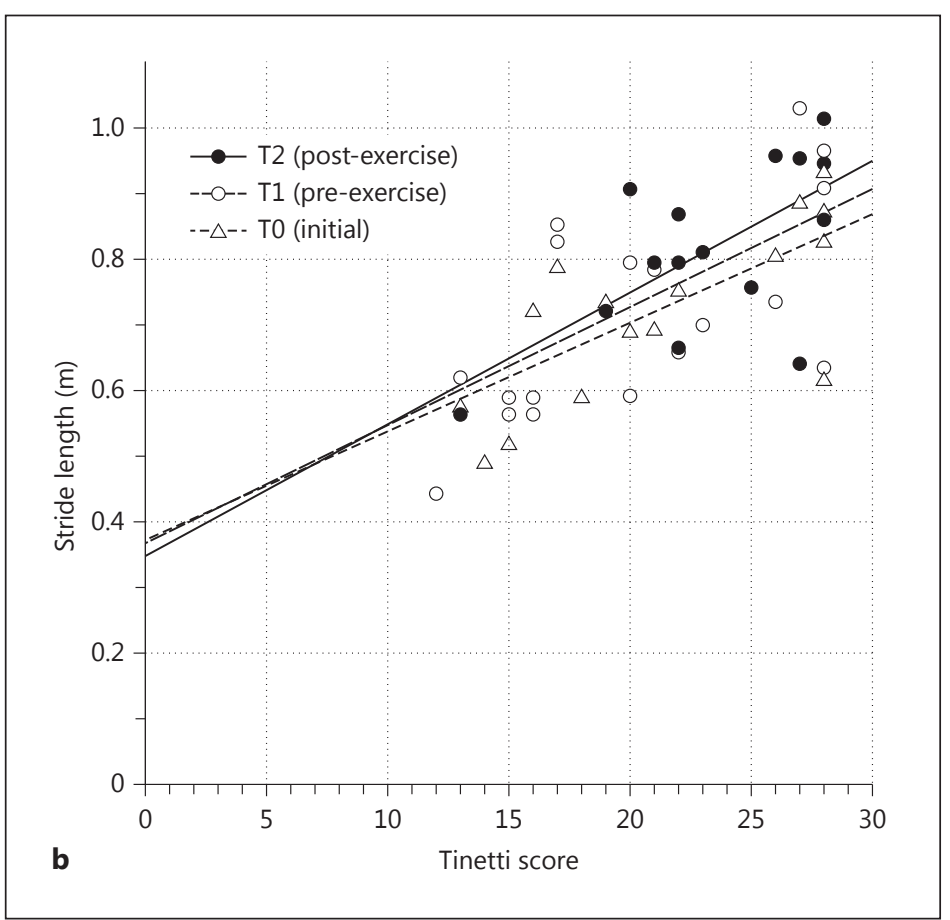

al. [11] who indicated that multicomponent intervention contributes to enhancing posture and balance, thereby improving the Tinetti score. Therefore, clinical tests such as the Tinetti scale [11] and the Timed Up and Go [12] are suitable to assess the effects of an exercise program on balance capabilities in dementia patients.

The originality of this study lies in the fact that each patient constituted his/her own control and that we assessed the gait performance and risk of falling during a non-inter- 
Perrochon et al.: Effects of a Multicomponent Exercise Program on Spatiotemporal Gait Parameters, Risk of Falling and Physical Activity in Dementia Patients

vention and an intervention period. We noticed a significant difference in the gait analysis and the Tinetti test score after exercise (intervention period), whereas these variables were stabilized before beginning this intervention. Indeed, no changes in gait speed were observed between the initial measurement (T0) and the pre-exercise measurement (T1) (non-intervention period). The potential causes for the effectiveness of the exercise program are related to its specific characteristics. In fact, as in this study, most of the effective high-quality studies used an exercise program that combined various types of exercises including resistance, flexibility, walking and balance/coordination exercises $[5-7,18]$ as well as dual-task exercises [5]. In this study, we alternated between individual and group-based exercises in institutionalized settings. Thus, patients received a personalized exercise program with appropriate exercises, including specific walking exercises. In the studies resulting in a positive outcome, the frequency of intervention was at least bi-weekly over 3 months, and usually the intensity of the program increased progressively $[5,18]$. Our exercise program was shorter (8 weeks) than the usual ones, but generated positive effects on gait parameters and walking too. In our study, all participants finished the program, completing an average of $81 \%$ of the prescribed sessions with no adverse events reported.

We also observed that physical activity did not change the metabolic cost of each patient $(1.11 \pm 1.15$ MET for non-intervention vs. $1.15 \pm 0.22$ MET for intervention; $p>0.05)$. Patients may have adapted their activity level according to the type of exercises proposed during the 8-week multicomponent exercise program. Consequently, each patient's physical activity level was similar between the periods with and without intervention. A qualitative approach with a regular and personalized program may explain the positives effects on their gait performances.

\section{Relationship between Gait Parameters and the Tinetti Score}

This study also explored the potential link between gait parameters and the risk of falling. In the literature, many authors significantly associate the risk of falling and gait parameters in dementia patients [2,3]. Globally, impairments in temporal and spatial gait parameters have been reported among individuals in the early stages of Alzheimer's disease [23, 24, 29]. More precisely, examination of individual gait parameters in dementia patients has indicated that these parameters were significantly modified in multiple fallers [30, 31]. Indeed, they walked more slowly [30, 31], had shorter stride length [30, 31], spent longer time in double support [30], had a wider support width and showed more variability in stride length and swing time [30]. However, the literature describes non-faller dementia patients with a gait speed $>1.0 \mathrm{~m} / \mathrm{s}[23,24,29]$, whereas faller dementia patients have a gait speed ranging from 0.62 to $0.78 \mathrm{~m} / \mathrm{s}$ according to the stage of dementia [4,30,31]. In our population, patients had low gait performances (gait speed $<0.8 \mathrm{~m} / \mathrm{s}$ ) correlated with the Tinetti score (T0, T1 and T2). It seems that analysis of gait speed in the single walking task can be used to detect moderate to severe dementia patients at risk of falling [31], whereas this parameter is used less in the literature [3]. While Locométrix ${ }^{\circledR}$ remains a simple and fast gait analysis system for clinical use, we proposed in this study a deep analysis of the gait to estimate the effects of the multicomponent exercise program and the risk of falling.

\section{Limitations}

Some limitations of this study need to be considered. First, the exercise program improved gait speed, but its value was still low (gait speed $<0.8 \mathrm{~m} / \mathrm{s}$ ) and was always associated with the risk of adverse outcomes after interventions [32]. Likewise, we observed an improvement in the Tinetti score for participants who had a high risk of falling, but most participants still had a low risk of falling. These results are probably associated with a very low gait performance related to age ( $86.7 \pm 5.4$ years) and the stage of dementia as well as the fact that several participants (8 patients) were multiple fallers before the exercise program. 
Perrochon et al.: Effects of a Multicomponent Exercise Program on Spatiotemporal Gait Parameters, Risk of Falling and Physical Activity in Dementia Patients

Second, like a number of studies [33], this study included few participants and has weak power. Several studies have failed to accurately diagnose their patients as having dementia. In many studies, the definition of dementia relied only on the MMSE score, which is not enough to justify a diagnosis of dementia. Nevertheless, 4 participants in this study were recruited from their MMSE scores only.

Finally, an accelerometer such as Locométrix ${ }^{\circledR}$ is a high-performance tool for defining a specific gait pattern for each cognitive profile $[23,24]$ and is an efficient method [20,22]. However, gait parameters analysis and interpretation are difficult with this technology. Although stride time variability is becoming a relevant marker of risk of falling [3], it is difficult to use regularity parameter in this study because it is only an index and not an absolute value.

\section{Perspectives}

Gait and the risk of falling are associated with frontal cognitive functions at different stages of Alzheimer's disease [4]. Executive and attention dysfunctions have been suggested to be the main cause of these early gait impairments [34], which may become subsequent risk factors of falling [35]. Several studies have demonstrated the positive effect of a multicomponent exercise program on cognitive functions $[9,33,36]$. In this study we did not assess the executive and attention functions capabilities of our dementia patients; nevertheless, these seem to improve through exercise programs.

In order to obtain optimal results in future studies, we recommend that authors include a second non-intervention period at the end of a multicomponent exercise program to assess the maintenance of the improvements.

\section{Conclusion}

A short-term, 8-week multicomponent exercise program including walking and balance exercises, functional exercises, muscle strengthening and flexibility improved gait performance and reduced the risk of falling in dementia patients most at risk. Assessment of spatiotemporal gait parameters using an accelerometer method (Locométrix ${ }^{\circledR}$ ) provided a quick and easy tool to estimate the benefits of an exercise program on gait performance.

\section{Disclosure Statement}

The authors of this paper have no conflict of interest in this work.

\section{References}

$\checkmark 1$ Scherder E, Eggermont L, Swaab D, van Heuvelen M, Kamsma Y, de Greef M, van Wijck R, Mulder T: Gait in ageing and associated dementias; its relationship with cognition. Neurosci Biobehav Rev 2007;31:485-497.

2 Beauchet O, Allali G, Berrut G, Hommet C, Dubost V, Assal F: Gait analysis in demented subjects: interests and perspectives. Neuropsychiatr Dis Treat 2008;4:155-160.

3 Montero-Odasso M, Verghese J, Beauchet O, Hausdorff JM: Gait and cognition: a complementary approach to understanding brain function and the risk of falling. J Am Geriatr Soc 2012;60:2127-2136.

-4 Coelho FG, Stella F, de Andrade LP, Barbieri FA, Santos-Galduroz RF, Gobbi S, Costa JL, Gobbi LT: Gait and risk of falls associated with frontal cognitive functions at different stages of Alzheimer's disease. Neuropsychol Dev Cogn B Aging Neuropsychol Cogn 2012;19:644-656.

-5 Schwenk M, Zieschang T, Oster P, Hauer K: Dual-task performances can be improved in patients with dementia: a randomized controlled trial. Neurology 2010;74:1961-1968. 
-6 Shaw FE, Bond J, Richardson DA, Dawson P, Steen IN, McKeith IG, Kenny RA: Multifactorial intervention after a fall in older people with cognitive impairment and dementia presenting to the accident and emergency department: randomised controlled trial. BMJ 2003;326:73.

7 Toulotte C, Fabre C, Dangremont B, Lensel G, Thevenon A: Effects of physical training on the physical capacity of frail, demented patients with a history of falling: a randomised controlled trial. Age Ageing 2003;32:67-73.

8 Doi T, Makizako H, Shimada H, Yoshida D, Tsutsumimoto K, Sawa R, Misu S, Suzuki T: Effects of multicomponent exercise on spatial-temporal gait parameters among the elderly with amnestic mild cognitive impairment (aMCI): preliminary results from a randomized controlled trial (RCT). Arch Gerontol Geriatr 2013;56:104-108.

-9 Kemoun G, Thibaud M, Roumagne N, Carette P, Albinet C, Toussaint L, Paccalin M, Dugue B: Effects of a physical training programme on cognitive function and walking efficiency in elderly persons with dementia. Dement Geriatr Cogn Disord 2010;29:109-114.

-10 Schwenk M, Zieschang T, Englert S, Grewal G, Najafi B, Hauer K: Improvements in gait characteristics after intensive resistance and functional training in people with dementia: a randomised controlled trial. BMC Geriatr 2014;14:73.

11 Santana-Sosa E, Barriopedro MI, Lopez-Mojares LM, Perez M, Lucia A: Exercise training is beneficial for Alzheimer's patients. Int J Sports Med 2008;29:845-850.

12 Suttanon P, Hill KD, Said CM, Williams SB, Byrne KN, LoGiudice D, Lautenschlager NT, Dodd KJ: Feasibility, safety and preliminary evidence of the effectiveness of a home-based exercise programme for older people with Alzheimer's disease: a pilot randomized controlled trial. Clin Rehabil 2013;27:427-438.

13 Potter R, Ellard D, Rees K, Thorogood M: A systematic review of the effects of physical activity on physical functioning, quality of life and depression in older people with dementia. Int J Geriatr Psychiatry 2011;26: 1000-1011.

14 Gillespie LD, Robertson MC, Gillespie WJ, Sherrington C, Gates S, Clemson LM, Lamb SE: Interventions for preventing falls in older people living in the community. Cochrane Database Syst Rev 2012;9:CD007146.

-15 Howe TE, Rochester L, Neil F, Skelton DA, Ballinger C: Exercise for improving balance in older people. Cochrane Database Syst Rev 2011;11:CD004963.

16 Martin JT, Wolf A, Moore JL, Rolenz E, DiNinno A, Reneker JC: The effectiveness of physical therapist-administered group-based exercise on fall prevention: a systematic review of randomized controlled trials. J Geriatr Phys Ther 2013;36:182-193.

17 Hauer K, Becker C, Lindemann U, Beyer N: Effectiveness of physical training on motor performance and fall prevention in cognitively impaired older persons: a systematic review. Am J Phys Med Rehabil 2006;85:847857.

18 Rolland Y, Pillard F, Klapouszczak A, Reynish E, Thomas D, Andrieu S, Riviere D, Vellas B: Exercise program for nursing home residents with Alzheimer's disease: a 1-year randomized, controlled trial. J Am Geriatr Soc 2007;55:158-165.

19 Bridenbaugh SA, Kressig RW: Laboratory review: the role of gait analysis in seniors' mobility and fall prevention. Gerontology 2011;57:256-264.

20 Gillain S, Petermans J: Contribution of new techniques to study the gait in old populations. Ann Phys Rehabil Med 2013;56:384-395.

21 Auvinet B, Berrut G, Touzard C, Moutel L, Collet N, Chaleil D, Barrey E: Reference data for normal subjects obtained with an accelerometric device. Gait Posture 2002;16:124-134.

22 Bautmans I, Jansen B, Van Keymolen B, Mets T: Reliability and clinical correlates of 3D-accelerometry based gait analysis outcomes according to age and fall-risk. Gait Posture 2011;33:366-372.

-23 Maquet D, Lekeu F, Warzee E, Gillain S, Wojtasik V, Salmon E, Petermans J, Croisier JL: Gait analysis in elderly adult patients with mild cognitive impairment and patients with mild Alzheimer's disease: simple versus dual task: a preliminary report. Clin Physiol Funct Imaging 2010;30:51-56.

24 Gillain S, Warzee E, Lekeu F, Wojtasik V, Maquet D, Croisier JL, Salmon E, Petermans J: The value of instrumental gait analysis in elderly healthy, MCI or Alzheimer's disease subjects and a comparison with other clinical tests used in single and dual-task conditions. Ann Phys Rehabil Med 2009;52:453-474.

25 Mignardot JB, Deschamps T, Barrey E, Auvinet B, Berrut G, Cornu C, Constans T, de Decker L: Gait disturbances as specific predictive markers of the first fall onset in elderly people: a two-year prospective observational study. Front Aging Neurosci 2014;6:22.

26 Folstein MF, Folstein SE, McHugh PR: 'Mini-mental state'. A practical method for grading the cognitive state of patients for the clinician. J Psychiatr Res 1975;12:189-198.

27 Tinetti ME, Williams TF, Mayewski R: Fall risk index for elderly patients based on number of chronic disabilities. Am J Med 1986;80:429-434.

-28 Oshima Y, Kawaguchi K, Tanaka S, Ohkawara K, Hikihara Y, Ishikawa-Takata K, Tabata I: Classifying household and locomotive activities using a triaxial accelerometer. Gait Posture 2010;31:370-374.

29 Nadkarni NK, Mawji E, McIlroy WE, Black SE: Spatial and temporal gait parameters in Alzheimer's disease and aging. Gait Posture 2009;30:452-454.

-30 Taylor ME, Delbaere K, Mikolaizak AS, Lord SR, Close JC: Gait parameter risk factors for falls under simple and dual task conditions in cognitively impaired older people. Gait Posture 2013;37:126-130.

-31 Sterke CS, van Beeck EF, Looman CW, Kressig RW, van der Cammen TJ: An electronic walkway can predict short-term fall risk in nursing home residents with dementia. Gait Posture 2012;36:95-101. 
32 Abellan van Kan G, Rolland Y, Andrieu S, Bauer J, Beauchet O, Bonnefoy M, Cesari M, Donini LM, Gillette Guyonnet S, Inzitari M, Nourhashemi F, Onder G, Ritz P, Salva A, Visser M, Vellas B: Gait speed at usual pace as a predictor of adverse outcomes in community-dwelling older people an International Academy on Nutrition and Aging (IANA) Task Force. J Nutr Health Aging 2009;13:881-889.

33 Pitkala K, Savikko N, Poysti M, Strandberg T, Laakkonen ML: Efficacy of physical exercise intervention on mobility and physical functioning in older people with dementia: a systematic review. Exp Gerontol 2013;48: 85-93.

34 Perrochon A, Kemoun G, Watelain E, Berthoz A: Walking Stroop carpet: an innovative dual-task concept for detecting cognitive impairment. Clin Interv Aging 2013;8:317-328.

35 Yogev-Seligmann G, Hausdorff JM, Giladi N: The role of executive function and attention in gait. Mov Disord 2008;23:329-342; quiz 472.

-36 Ohman H, Savikko N, Strandberg TE, Pitkala KH: Effect of physical exercise on cognitive performance in older adults with mild cognitive impairment or dementia: a systematic review. Dement Geriatr Cogn Disord 2014; $38: 347-365$. 